\title{
ALTEC y la Gestión Tecnológica en Iberoamérica: Investigación y Gestión en las Empresas
}

\author{
Domingo González', Roberto Sbragia², Oscar Galante², Rocío Soto ${ }^{4}$, Renato Valdivieso ${ }^{5}$
}

\begin{abstract}
The seminars of the Latin Ibero-American Association of Technology Management (ALTEC) they are one of the most important spaces in this region for the discussion of the research in the discipline of innovation and technology management. This article presents a brief panorama of the main thematic areas approached in 14 seminars carried out by that association from 1985 until the year 201I. In this context, a group of 18 articles is presented, which discuss in this volume the technology and innovation management in Latin American companies of diverse size, from an individual and sectoral focus.
\end{abstract}

Keywords: innovation and technology management; new enterprises.

Los encuentros bianuales realizados por la Asociación Latino-Iberoamericana de Gestión Tecnológica (ALTEC) constituyen en la actualidad uno de los espacios más importantes en esta región para la discusión de la investigación en la disciplina de la gestión de la innovación y la tecnología. En este artículo se presenta brevemente un panorama de las principales áreas temáticas abordadas en 14 encuentros realizados por esa asociación desde 1985 hasta el año 20II. En este contexto, se presenta un conjunto de 18 artículos que tratan en este volumen sobre la gestión de la innovación y la tecnología en empresas latinoamericanas de diverso tamaño, desde un enfoque individual y sectorial.

Keywords: gestión de la innovación y la tecnología; nuevas empresas.

I Departamento de Ingeniería, Pontificia Universidad Católica del Perú. Av. Universitaria I80I, San Miguel, Lima 32, Perú. Telefono: +51.1.6262000. Contacto principal: dgonzal@pucp.edu.pe

${ }^{2}$ Departamento de Administração, Universidade de São Paulo. Av. Prof. Luciano Gualberto, 908, São Paulo, CEP: 05508-0 I0, Brasil.

${ }^{3}$ Instituto Nacional de Tecnología Industrial. Colectora de Avenida General Paz 5445, Casilla de correo I57, BI650KNA. San Martín, República Argentina.

${ }^{4}$ Instituto Politécnico Nacional (IPN). Av. Luis Enrique Erro S/N, Unidad Profesional Adolfo López Mateos, Zacatenco, Delegación Gustavo A. Madero, C.P. 07738, México, Distrito Federal, 2009-20I0.

${ }^{5}$ Fundación Empresas Polar. Segunda avenida, Los Cortijos de Lourdes, Edificio Fundación Empresas Polar, I Piso. Apartado postal 70934. Los Ruices. Zona postal: I07I-A. Caracas. Venezuela.

ISSN: 07 I8-2724. (http://www.jotmi.org)

Journal of Technology Management \& Innovation (c) Universidad Alberto Hurtado, Facultad de Economía y Negocios. 


\section{Introducción}

La Asociación Latino-lberoamericana de Gestión Tecnológica - ALTEC, fue fundada en octubre de 1984 con el propósito de vincular personas, físicas y jurídicas, activas en la reflexión y el ejercicio de la gestión tecnológica para realizar actividades de cooperación en dicha área. La asociación está integrada por un grupo significativo de profesionales de la región interesados en la política y gestión de la innovación y la tecnología, incluyendo entre sus más de 500 asociados a investigadores, profesores universitarios, empresarios y profesionales de las empresas, especialistas gubernamentales, expertos de agencias financieras y de cooperación y profesionales que se desempeñan en consultoras privadas.

Los orígenes de la asociación se encuentran en la Reunión Latinoamericana de Gestión de la Innovación Tecnológica promovida por la Organización de los Estados Americanos (OEA) y el Gobierno del Brasil, la cual se realizó octubre de 1983, en las instalaciones de la Universidade de São Paulo en Brasil. En ese encuentro se examinó el estado del arte sobre la innovación tecnológica en América Latina y se propusieron mecanismos y medidas para su dinamización, con énfasis en la integración del sector productivo con los esfuerzos de investigación realizados en institutos especializados de la infraestructura científica - tecnológica y en las universidades (Galante y Pérez, 2008).

La favorable experiencia de la reunión estimuló a multiplicar las observaciones, análisis y conclusiones de los diversos problemas del desarrollo científico y tecnológico de los países de la región latinoamericana. Los resultados conseguidos en este ejercicio y en otros similares indicaron la conveniencia de institucionalizar los intercambios, encuentros y colaboración entre los diversos centros de estudios y de capacitación establecidos en los países latinoamericanos, para la solución de los diversos problemas de la administración de la ciencia y tecnología. A partir de esta conclusiones, se propuso la creación de una asociación flexible integrada por personas e instituciones interesadas, a fin de enfrentar mejor la agenda del problema que comprendía cuestiones de complejidad creciente, como eran el financiamiento de la ciencia y tecnología, el avance las industrias basadas en la ciencia, el avance en la ciencia y tecnología mismas y la vinculación de las políticas y planes de la industria y tecnología en los niveles macro y microeconómico, entre otros.

Sobre estas bases, se funda la asociación y en septiembre de 1985 se realizó el Primer Seminario Latinoamericano ALTEC en la Universidade de São Paulo. En ella los socios debatieron, aprobaron los estatutos y acordaron las prioridades de la asociación durante los dos años siguientes. A partir de ese momento, cada dos años, de manera ininterrumpida, se realizan los encuentros de la asociación denominados ini- cialmente Seminarios y más adelante Congresos de Gestión Tecnológica. Con el tiempo los encuentros de ALTEC se han constituido como el evento académico más importante de la disciplina en Iberoamérica.

\section{El panorama de la Gestión Tecnológica en los encuentros de ALTEC}

Desde su creación, la asociación ALTEC ha realizado 14 encuentros académicos, los cuales se iniciaron con una estructura de seminario hasta constituirse en las últimas ediciones en congresos de gran magnitud. La Tabla I muestra la evolución de las ponencias presentadas en los encuentros, alcanzando casi 3000 ponencias en total desde 1985 hasta el año $201 \mathrm{l}$.

Desde sus orígenes, los encuentros de ALTEC han tratado los varios niveles de la gestión de la tecnología y la innovación, desde los aspectos al nivel de los proyectos y las empresas, pasando por los espacios interorganizacionales y de territorios donde confluyen diversos actores, hasta los aspectos contextuales y de construcción de entornos favorables al desarrollo de la ciencia, la tecnología y la innovación. Del mismo modo, a lo largo del tiempo, los encuentros de ALTEC han venido incorporando los nuevos enfoques $y$ conceptos y los temas de actualidad en la disciplina. La denominación de las áreas temáticas ha variado a lo largo del tiempo, sin embargo, de manera general se han abordado las grandes áreas temáticas indicadas en la Tabla 2.

\begin{tabular}{|l|l|l|l|}
\hline Año & País & Ciudad sede & $\begin{array}{l}\text { Número de ponencias } \\
\text { presentadas }\end{array}$ \\
\hline 1985 & Brasil & Sao Paulo & 26 \\
\hline 1987 & México & México D.F. & 76 \\
\hline 1989 & Argentina & Buenos Aires & 92 \\
\hline 1991 & Venezuela & Caracas & 84 \\
\hline 1993 & Colombia & Bogotá & 53 \\
\hline 1995 & Chile & Concepción & 102 \\
\hline 1997 & Cuba & La Habana & 193 \\
\hline 1999 & España & Valencia & 256 \\
\hline 2001 & Costa Rica & San José & 196 \\
\hline 2003 & México & México D.F. & 274 \\
\hline 2005 & Brasil & Salvador & 392 \\
\hline 2007 & Argentina & Buenos Aires & 348 \\
\hline 2009 & Colombia & Cartagena & 327 \\
\hline 2011 & Perú & Lima & 514 \\
\hline Total & 2933 & & \\
\hline
\end{tabular}

Tabla I. Encuentros ALTEC 1985 - 201 I.

Fuente:ALTEC (1985; 1987; 1989; 1991; 1993; 1995; 1997; 1999; $200 \mathrm{I} ; 2003 ; 2005 ; 2007 ; 2009 ; 20$ II)

ISSN: 07 I8-2724. (http://www.jotmi.org)

Journal of Technology Management \& Innovation (c) Universidad Alberto Hurtado, Facultad de Economía y Negocios. 
Algunas de las áreas temáticas abordadas en los catorce encuentros se han mantenido, indicando el permanente interés en la región por los temas vinculados al entorno de los sistemas de innovación y a la construcción de ambientes favorables para el desarrollo de las ciencia, la tecnología y la innovación; a la gestión de la tecnología y la innovación en la empresa; a la política científica y tecnológica y a la gestión de la I+D. Del mismo modo, se observa la emergencia de temas específicos tales como la dinámica de la creación de empresas innovadoras, la gestión ambiental y del conocimiento, entre otros. La Tabla 4 muestra el porcentaje de ponencias presentadas desde 1985 hasta 201 I en cada área temática, indicando el grado de interés en la región en cada tema.
Quirino et al. (200I), Sbragia et al. (2003) han estudiado en años pasados las características de los encuentros ALTEC en los temas y número de trabajos presentados, sistema de autoría, distribución de las ponencias por país, por instituciones y por autores con mayor contribución de ponencias a los encuentros. En aquella época los autores vislumbraron las tendencias hoy consolidadas, tales como el creciente interés en la disciplina, las preferencias por las áreas temáticas indicadas anteriormente $y$ el aumento de la coautoría en las ponencias presentadas en los encuentros.

\begin{tabular}{|c|c|}
\hline Áreas temáticas & Contenido \\
\hline $\begin{array}{l}\text { La Vinculación entre los acto- } \\
\text { res para la innovación: }\end{array}$ & $\begin{array}{l}\text { Las relaciones entre las actividades de I+D de la academia y el sector productivo; la relación universidad-empresa; } \\
\text { las estructuras organizacionales de interfaz; cooperación de la industria con entidades del estado; la transferencia } \\
\text { de los resultados de la I+D. }\end{array}$ \\
\hline $\begin{array}{l}\text { Gestión de la innovación y la } \\
\text { tecnológica en la empresa }\end{array}$ & $\begin{array}{l}\text { Gestión en empresas innovadoras; experiencias de gestión de la tecnología en sectores específicos; gestión de la } \\
\text { innovación de la micro, pequeña y mediana empresa; alianzas y cooperación interempresarial en proyectos de in- } \\
\text { novación, nuevas tecnologías de producción y sistemas de información; cultura organizacional para la innovación; } \\
\text { planeamiento estratégico de la tecnologí; perfil de los gestores de innovación; propiedad industrial e intelectual; } \\
\text { prospectiva y vigilancia tecnológica; gestión tecnológica en nuevos modelos de organización empresarial. }\end{array}$ \\
\hline Sistemas de innovación & $\begin{array}{l}\text { Sistemas sectoriales, regionales, nacionales y locales de innovación; redes de investigación e innovación; la } \\
\text { construcción de entornos favorables para la innovación; organización regional, nacional y estatal para la gestión } \\
\text { en ciencia y tecnología; estrategias locales y regionales de innovación tecnológica; clusters, arreglos productivos, } \\
\text { territorio y desarrollo local, territorio e industria creativa; competitividad sistémica, industrial y empresarial; } \\
\text { competitividad, empleo y desarrollo sostenible; normas técnicas y otros aspectos de la regulación de la ciencia y } \\
\text { la tecnología. }\end{array}$ \\
\hline Gestión de la I+D & $\begin{array}{l}\text { La función de I+D en la empresa; gestión de riesgo y técnicas de planeamiento, control y evaluación; organización } \\
\text { de equipos de proyectos; portafolio de proyectos; instrumentos de promoción de la I+D; política y gestión de } \\
\text { investigación e innovación tecnológica en universidades e institutos de investigación; evaluación del desempeño } \\
\text { en instituciones de investigación. }\end{array}$ \\
\hline Política científica y tecnológica & $\begin{array}{l}\text { Políticas, fuentes e instrumentos de financiamiento de la innovación tecnológica; aspectos jurídicos de la } \\
\text { innovación; política de innovación: programas y redes internacionales; Integración latinoamericana y gestión } \\
\text { tecnológica; incentivos fiscales, no fiscales y otros mecanismos inductores de innovación; los impactos sociales, } \\
\text { económicos y ambientales de la innovación; indicadores de ciencia, tecnología e innovación; evaluación en I+D e } \\
\text { innovación. }\end{array}$ \\
\hline $\begin{array}{l}\text { Creación de empresas innova- } \\
\text { doras y de base tecnológica, } \\
\text { incubadoras, polos y parques }\end{array}$ & $\begin{array}{l}\text { Emprendedores y generación de empresas de base tecnológica; financiamiento de nuevas empresas; planeamiento } \\
\text { y gestión de incubadoras, polos y parques tecnológicos. }\end{array}$ \\
\hline Gestión del Conocimiento & $\begin{array}{l}\text { La innovación y la gestión del conocimiento; generación, gestión y transferencia del conocimiento; indicadores y } \\
\text { gestión del conocimiento; gestión del conocimiento y de la información, calidad y productividad. }\end{array}$ \\
\hline Ciencia, Tecnología y Sociedad & $\begin{array}{l}\text { Aspectos sociales y culturales de la gestión tecnológica; innovación, ética y sociedad; economía solidaria e } \\
\text { innovación tecnológica; estudios de género y tecnología; tecnología y gestión urbana; tecnología social; innovación } \\
\text { social. }\end{array}$ \\
\hline Formación de capacidades & $\begin{array}{l}\text { Formación de recursos humanos en gestión tecnológica; formación para el desarrollo de la capacidad innovadora; } \\
\text { Tecnología, formación y empleabilidad; estrategias de aprendizaje y construcción de capacidades de innovación. }\end{array}$ \\
\hline $\begin{array}{l}\text { Desarrollo sustentable y } \\
\text { gestión ambiental }\end{array}$ & $\begin{array}{l}\text { Relaciones entre desarrollo tecnológico y medio ambiente; aspectos ambientales de la innovación tecnológica; } \\
\text { Gestión tecnológica para el desarrollo sustentable; gestión ambiental. }\end{array}$ \\
\hline $\begin{array}{l}\text { Herramientas de apoyo a la } \\
\text { gestión de la I+D e innovación }\end{array}$ & $\begin{array}{l}\text { Aplicación de técnicas específicas cualitativas y cuantitativas; propuesta, desarrollo y aplicación de métodos y } \\
\text { metodologías para la gestión de la I+D e innovación, }\end{array}$ \\
\hline Temas emergentes & $\begin{array}{l}\text { Nuevos desarrollos y conceptos en política de ciencia tecnología e innovación; nuevos conceptos, metodologías y } \\
\text { técnicas en la gestión de la innovación y la tecnología. }\end{array}$ \\
\hline
\end{tabular}

Tabla 2. Áreas temáticas de los encuentros ALTEC.

Fuente:ALTEC (1985; 1987; 1989; 199I; 1993; 1995; 1997; 1999; 200I; 2003; 2005; 2007; 2009; 20II)

ISSN: 07 I8-2724. (http://www.jotmi.org)

Journal of Technology Management \& Innovation (c) Universidad Alberto Hurtado, Facultad de Economía y Negocios. 


\section{La Edición Especial}

En este volumen se presentan 18 artículos que abordan la gestión de la innovación y la tecnología en empresas de diferentes tamaños, áreas de actuación y fases de desarrollo, desde un enfoque de la empresa individual y también desde un punto de vista sectorial.

Loa primeros 4 artículos tratan de investigaciones que indagan sobre la gestión de la innovación y la tecnología en empresas de gran tamaño. Así, Ades et al. (20I2) analizan tres casos de empresas brasileñas, cuyos procesos de gestión de la innovación se encuentran consolidados. Los autores estudian a las empresas Natura, IBM (filial Brasil) y Siemens (ChemTech/Brasil) con el objetivo de analizar como ocurrió la implementación de la innovación abierta. La investigación concluye que el proceso de la implementación de la innovación abierta se encuentra en fase inicial en los tres casos estudiados y ocurre de manera paralela a las inversiones en innovación cerrada, siendo prematuro aún para observar resultados de la innovación abierta en esas empresas. Por otro lado, Jugend et al. (20l2) abordan el tema del diseño de nuevos productos y las prácticas de integración que se dan en este contexto entre el área de I+D y las otras áreas de la empresa involucradazas en el proceso. Para ello los autores realizan dos estudios de caso en los cuales encuentran que existen prácticas favorables al proceso de diseño de productos tales como la participación del personal de I+D en actividades de investigación de mercados, el uso de mecanismos virtuales de interacción y la aplicación de métodos como mapas tecnológicos. Assalim y Almeida (2012) estudian la forma en que la evaluación de conformidad en grandes proyectos de ingeniería puede ser utilizada como herramienta de aprendizaje organizacional por las entidades involucradas en el proyecto. Con esta finalidad, los autores realizan un estudio de caso en la empresa Petrobras, abordando varios proyectos de ingeniería y concluyen que el modelo conceptual propuesto es un instrumento importante de gestión y de aprendizaje organizacional. Finalmente, Oliveira et al. (2012) analizan y evalúan la metodología para la constitución de asociaciones en redes denominada Práctica Centros y Redes de Excelencia (PCREX) implementada por la empresa Petrobrás. Con esta finalidad los autores estudian 8 centros constituidos en Brasil con esta metodología, la cual es contrastada con modelos conceptuales más recientes como la innovación abierta y se verifica su compatibilidad. La investigación también concluye que la metodología puede ser usada para enfrentar desafíos y problemas cuyas soluciones dependen de conjugar el conocimiento disperso entre la empresa y universidades, clientes, proveedores y competidores.

\section{Con el foco en las pequeñas y medianas empresas}

El segundo conjunto de artículos presentados en este volumen aborda 5 investigaciones sobre la gestión de la innovación y la tecnología en pequeñas y medianas empresas en operación o en proceso de creación. Inicialmente, Alves et al. (20I2) estudian dos redes de pequeñas y medianas empresas minoristas del sector de supermercados en el estado de Minas Gerais en Brasil, las cuales implementan estrategias colectivas para enfrentar la competencia. En ese proceso, los autores evalúan e identifican innovaciones organizacionales, innovaciones en servicios e innovaciones procesos en estas empresas que son consecuencia de su acción colectiva. Por otro lado, Yrigoyen (2012) explora el fenómeno de innovación, desde una perspectiva multidimensional, en dos grupos de micro y pequeñas empresas de sectores tradi-

\begin{tabular}{|l|l|}
\hline Área Temática & Ponencias (\%) \\
\hline Sistemas de innovación & 19.8 \\
\hline Gestión tecnológica en la empresa & 19.7 \\
\hline Vinculación entre los actores & $\mathrm{Il} .4$ \\
\hline Gestión del conocimiento & $\mathrm{II} . \mathrm{I}$ \\
\hline Creación de empresas innovadoras y de base tecnológica, incubadoras, polos y parques & 7.1 \\
\hline Gestión de la I+D & 6.3 \\
\hline Ciencia, tecnología y sociedad & 6.0 \\
\hline Política científica y tecnológica & 5.2 \\
\hline Herramientas de apoyo a la gestión de la I+D e innovación & 4.9 \\
\hline Desarrollo sustentable y gestión ambiental & 4.2 \\
\hline Formación de capacidades & 3.0 \\
\hline Temas emergentes & 1.3 \\
\hline Total & 100.0 \\
\hline
\end{tabular}

Tabla 4. Ponencias por áreas temáticas de los encuentros ALTEC (I985 - 20I I) Fuente:ALTEC (1985; 1987; 1989; 199I; 1993; 1995; 1997; 1999; 200I; 2003; 2005; 2007; 2009; 20I I)

ISSN: 07I 8-2724. (http://www.jotmi.org)

Journal of Technology Management \& Innovation (c) Universidad Alberto Hurtado, Facultad de Economía y Negocios. 
cionales en Perú, constituidos por 37 empresas del sector de carpintería y manufactura de muebles y 13 empresas del sector de producción de pisco. El autor analiza innovaciones orientadas a la demanda tales como innovaciones de producto, mercadotecnia y mercado, así como también innovaciones orientadas a la eficiencia operacional o reducción de costos las cuales incluyen innovaciones de procesos de producción e innovaciones de procesos administrativos. El trabajo concluye en que los dos grupos de empresas difieren en la intensidad con que practican las innovaciones orientadas a la demanda, mientras que presentan un comportamiento similar en cuanto a la intensidad con que practican las innovaciones orientadas a la eficiencia operacional o reducción de costos.

En el ámbito de la dinámica de la creación de nuevas empresas, Frick y Frick (20I2) presentan un método de gestión de una nueva empresa de base tecnológica, el cual está basado en la metodología denominada Capital Emprendedor Colaborativo desarrollada por un grupo de ángeles inversionistas que actúan en Brasil desde el año 2008. Los autores realizan un estudio de caso en la empresa start up Phacton del sector de tecnologías de información, la cual fue apoyada por ese grupo empleando el método mencionado. El trabajo concluye indicando que este método presenta resultados positivos para el desarrollo de una nueva empresa. Moraes, Lobosco y Lima (20I2) estudian el tema del financiamiento de nuevas empresas, indagando sobre las relaciones entre los financiadores ángeles inversionistas del grupo São Paulo Anjos (SPA) y la agencia FINEP del Brasil con las empresas financiadas. Los resultados indican que la expectativa para la FINEP en esta relación es que las empresas cumplan los plazos establecidos por la institución. Mientras tanto para SPA las expectativas de la relación son disponer del capital y tiempo en las inversiones seleccionadas y que los emprendedores tengan conocimiento de gestión y participen de redes de contactos técnicos. Finalmente, Ortiz y Pedroza (20/2) estudian la problemática de colisiones entre autos en la Zona Metropolitana de Guadalajara en México, haciendo un análisis morfológico para identificar las necesidades y las oportunidades tecnológicas que se presentan en este tema, como sustento para desarrollar el proyecto de una empresa de dispositivos electrónicos para la seguridad de vehículos. El análisis morfológico se realiza a través de la recopilación de documentos de patente del área tecnológica en estudio, extrayendo las dimensiones y las formas de las tecnologías disponibles para generar una matriz morfológica. El trabajo concluye proponiendo que el análisis morfológico basado en palabras clave en la búsqueda de patentes, se use como una herramienta de prospectiva tecnológica, cuyo objetivo es facilitar al experto el proceso inventivo.

Desde un enfoque sectorial, el tercer conjunto de artículos presentados en este volumen aborda 6 investigaciones so- bre la gestión de la innovación y la tecnología en sectores específicos. Gielfi et al. (20I2) abordan el sector de petróleo brasileño analizando la importancia de la interacción entre el usuario y el productor para el proceso de innovación. El estudio se enfoca en dos empresas proveedoras del conjunto de válvulas denominadas "árbol de navidad mojado", el cual es esencial en la exploración de petróleo en aguas profundas. Los autores concluyen que la industria de petróleo brasileña desarrolla una estrategia de innovación cooperativa, en la cual es muy importante la proximidad geográfica, especialmente entre los centros de ingeniería, investigación y desarrollo de las empresas.

Por otro lado, abordando el sector eléctrico brasileño, Moraes y Varela (20I2) proponen un modelo integrado de gestión de I+D desarrollado para el Grupo Eletrobrás en Brasil. Dicha iniciativa es consecuencia de la ley que obliga a las empresas del sector eléctrico a realizar inversiones en I+D y en programas de eficiencia energética. El modelo propuesto busca incorporar una visión de la gestión de la tecnología y de la innovación que esté alineada a las estrategias de negocios de las empresas del grupo y se nutre de buenas prácticas de gestión identificadas en el estudio en empresas de diversos sectores. Pinto y Botelho (20I2) presentan una metodología para la caracterización de proveedores del sector eléctrico brasileño con potencial para el desarrollo de productos innovadores. El trabajo aporta para el diseño de una estrategia de gestión de la innovación en las fases finales de la cadena de suministro, que permita incorporar a los proveedores de los concesionarios de distribución eléctrica en la actividad de I+D y alcanzar las metas del sector.

Pereira, Fujino y Silveira (20I2) estudian la innovación tecnológica del sector de equipamientos electromédicos analizando el universo de 169.756 registros de patentes del sector depositados mundialmente entre $196 \mathrm{I}$ y 2010 . Los autores presentan un panorama del desarrollo tecnológico del sector en América Latina, evaluando cuatro indicadores: total de registros en América Latina, evolución histórica de la producción, agentes productores, características del sector de equipamientos electromédicos. El trabajo concluye que el sector de equipamientos electromédicos en América Latina está en desarrollo, específicamente en la última década, con crecimiento exponencial en el número de depósitos de patentes, aunque su presencia no es aún significativa a novel mundial. Por otro lado, Hernández y González (20I2) enfocan su trabajo en el estudio de las tecnologías que sustentan la industria de la telefonía celular, a través de un estudio de caso de las empresas Nokia, Huawei y ZTE. Los autores analizan la actividad de patentamiento de estas empresas desde el $200 \mathrm{I}$ al $20 \mathrm{II}$, para obtener información sobre su actividad I+D en los últimos años buscando proporcionar una comprensión de la evolución tecnológica de la industria de celulares. El trabajo emplea el método 
estadístico del análisis de correspondencias para descubrir que las empresas mencionadas desarrollan su actividad I+D en las mismas líneas de investigación durantes los años estudiados. Finalmente, Agrasso Neto (2012) aborda la planificación estratégica de la comunicación para el Observatorio de Desarrollo Industrial de Santa Catarina. El trabajo es una investigación - acción que desarrolla un estudio prospectivo con la finalidad de generar conocimiento para apoyar la toma de decisiones en el sector de tecnologías de información del Estado de Santa Catarina en Brasil.

El último conjunto de artículos presentados en este volumen aborda 4 investigaciones sobre la gestión de la innovación y la tecnología en empresas de tecnologías de la información. Carlos Junior et al. (2012) abordan el tema de la computación en nube como un nuevo paradigma que hace posible el modelo de computación utilitaria, en que los recursos computacionales se ofrecen al consumidor como servicio sobre demanda. Los autores buscan identificar los aspectos de innovación asociados al modelo de computación en nube y su influencia en las estrategias de las empresas consumidoras de tecnología de información ( $\mathrm{Tl})$ y en el rol del profesional de $\mathrm{Tl}$ en las organizaciones. Con esta finalidad, realizan una encuesta entre los principales ejecutivos del área de TI de 64 empresas medianas y grandes con operaciones en Brasil. Los resultados indican que la adopción del modelo abre oportunidades de reducción de costos y aumento de la agilidad, lo cual influye en las empresas consumidoras a incluir computación en nube como estrategia. También, la investigación concluye que el área de $\mathrm{TI}$ de estas empresas gestiona servicios contratados de proveedores de computación en nube con el objetivo de optimizar procesos de negocios. Grise y Huertas (2012) estudian la implantación de herramientas de tecnologías de información, proponiendo un proceso de selección de ellas orientado a ofrecer un valor superior a los clientes en relaciones de negocios business to business. Con esta finalidad, los autores realizan una investigación exploratoria por medio de entrevistas en profundidad a dirigentes de tres empresas que usan herramientas de esta naturaleza. El proceso propuesto comienza por la comprensión del contexto de la empresa y el reconocimiento el estado inicial del valor ofrecido a los clientes $y$, sobre esta base, se procede con la intención estratégica que sustenta la selección y la implantación de las herramientas de TIC.

Correia et al. (2012) evalúan el grado de madurez en el uso de sistemas de información en 16 instituciones de salud del Estado de Santa Catarina en Brasil., evaluando el grado de madurez. Los autores concluyen que en las instituciones estudiadas existe una preocupación creciente por usar la información disponible para la toma de decisiones estratégicas, aunque los sistemas de las empresas estudiadas no presentan características para el análisis complejo de la información.
Finalmente, Grutzmann, Macedo y Zambalde (20/2) investigan aspectos del comportamiento del consumidor para aceptar productos innovadores, conjugando los conceptos de redes sociales, innovación y comportamiento del consumidor. El trabajo emplea una muestra de 127 alumnos de administración de una universidad federal brasileña e identifica que la gestión del conocimiento en las redes sociales puede ser usada para capturar informaciones que pueden contener críticas a productos y marcas o sugerencias de nuevos productos. Adicionalmente, los autores concluyen que las correlaciones encontradas en el estudio indican que existen consumidores con comportamiento innovador que actúan como difusores de nuevos productos y servicios. 


\section{Tabla de Contenidos}

ALTEC y la Gestión Tecnológica en Iberoamérica: Investigación y Gestión en las Empresas. Domingo González González, Roberto Sbragia, Oscar Galante, Rocío Soto, Renato Valdivieso

Implementing Open Innovation: The Case of Natura, IBM and Siemens. Cely Ades, Aline Figlioli, Roberto Sbragia, Geciane Porto, Guilherme Ary Plonski, Kleber Celadon

Integration Practices for the Technological Innovation of Products: Case Studies at Two Large Technological Companies. Daniel Jugend, Sérgio Luis da Silva, Luís Fernando Magnanini Almeida, José Alcides Gobbo Junior

Conformity Assessment as a Tool for Organizational Learning in Large Engineering and Construction Projects. Luciano Assalim, Maria Fatima Ludovico Almeida

Innovation in Innovation Management: The Experience of Petrobras Centers and Networks of Excellence Program. Ana Maria de Britto Pires, Francisco Lima Cruz Teixeira, Horacio Nelson Hastenreiter Filho, Sergio Ricardo Oliveira

Innovation Processes in Small and Medium Enterprises Associated in Networks from the Supermarket Sector. Rosália Rodrigues Alves, Mariane Figueira, Cleber Carvalho de Castro, Joel Yutaka Sugano, Ari de Souza Soares

Explorando Distintos Tipos de Innovación en Micro y Pequeñas Empresas Peruanas. José Isaac Yrigoyen

Gestión y Desarrollo de Empresas Innovadoras. Alexis Maximiliano Frick, Silvia Teresa Ferreira de Frick

Expectations of FINEP and São Paulo Anjos Agents Concerning the Use of Venture Capital in Technology-based Small and Medium Enterprises. Marcela Barbosa de Moraes, Antonio Lobosco, Edmilson Lima

Análisis Morfológico de Patentes para Desarrollar un Producto de Seguridad Vehicular. Sara Ortiz Cantú, Álvaro R. Pedroza Zapata, Esteban Martinez Guerrero

User-Producer Interaction in the Brazilian Oil Industry: The Relationship between Petrobras and its Suppliers of Wet Christmas Tree. Giovanna Guimarães Gielfi, Newton Müller Pereira, Rogério Gomes, Vinicius Cardoso de Barros Fornari

R,D\&I in Electric Sector: a Management Model. Edmilson Alves de Moraes, Carmen Augusta Varela 
Technological Innovation in the Electro-Medical Equipment Sector: Analysis in Latin America. Cesar Antonio Pereira, Asa Fujino, Marco Antonio Silveira

$140-149$

Strategic Communication Planning for the Industrial Development Observatory of Santa Catarina. Viviane D' Barsoles Gonçalves Werutsky, Paulo Maurício Selig, Fabrício Kurman Merlin, Vera Lúcia Duarte do Valle Pereira, Manoel Agrasso Neto

Estudio de la Evolución Tecnológica en Empresas de Celulares a Partir de la Actividad de Patentamiento. Carlos Hernández, Domingo González

Cloud Computing and Information Technology Strategy. Antonio Mariano Carlos Junior, Cesar Augusto Biancolino, Emerson Antonio Maccari

Choosing ICT Tools: Proposition of a Model to DeliverValue for Clients. Paulo Grise, Melby Karina Zuniga Huertas

The Use of Information Systems in Health Care Facilities: A Brazilian Case. Rion Brattig Correia, Jorge Chiodini, Oscar Dalfovo, Luiz Henrique Silva, Rosicler Teske

Knowledge Management and Innovation: The Role of Virtual Social Networks in Innovative Consumer Behavior. Andre Grutzmann, Fernanda Maria Felício Macedo, André Luiz Zambalde 


\section{Sobre los autores}

Domingo González es doctor en Ingeniería de Producción por la Pontificia Universidade Católica do Rio de Janeiro. Jefe del Departamento de Ingeniería y Director de la Maestría en Gestión y Política de la Innovación y la Tecnología de la Pontificia Universidad Católica del Perú. Presidente de la Asociación Latino-lberoamericana de Gestión Tecnológica (ALTEC).

Roberto Sbragia es graduado, magíster y doctor en administración por la Universidade de São Paulo. Ha realizado un posdoctorado en Management of R\&D por la Northwestern University/Technological Institute/Departament of Industrial Engineering and Management Sciences. Actualmente es profesor titular de la Universidade de São Paulo, en el Departamento de Administración de la FEA, donde coordina el grupo de estudios en Gestión de la Innovación y Proyectos Tecnológicos. Actúa principalmente en los siguientes temas: innovación, evaluación, industria, gestión de la innovación tecnológica y gestión de proyectos.

Oscar Galante es ingeniero de la UNAM- México, y Especialista en Política y Gestión de la Ciencia y la Tecnología de la Universidad del Salvador - Argentina. Investigador en temas de Vinculación Tecnológica. Docente de Posgrado de las universidades UBA, UNGS, UTN, UNSAM, entre otras. Ha sido Director de Relaciones con la Producción del CONICET, responsable de la Ley de Innovación del FONTAR, Director Nacional de Programas y Proyectos Especiales y Director Nacional de Desarrollo Tecnológico del MinCyT. Actualmente es Gerente de Asistencia Tecnológica para la Demanda Social del INTI; todas instituciones de CyT de Argentina. Miembro de ALTEC, INNRED y REDES. Ex-Presidente de ALTEC en dos períodos.

Rocío Soto es Doctora en Administración y Dirección de Empresas, por la Universidad Politécnica de Cataluña de España. Fue Directora del Centro de Investigaciones Económicas, Administrativas y Sociales del Instituto Politécnico Nacional de México (IPN). Investigadora de la Escuela Superior de Comercio y Administración unidad Sto. Tomás del IPN. Directora por México de ALTEC de octubre de 2009 a la fecha.

Renato Valdivieso es licenciado en Química, Msc. en Planificacion de Ciencia y Tecnología en Cendes-Universidad Central de Venezuela. Es Gerente de Proyectos (Jubilado) de la Fundación Empresas Polar. He sido profesor de posgrado en Cendes-UCV. Es Editor de la Revista Espacios y miembro de la directiva de Altec en los años 1991-1995 y 201 I-2013.

\section{Referencias Bibliográficas}

ALTEC. (20II). Anales del XIV Congreso Latino-lberoamericano de Gestión Tecnológica. ALTEC, Lima.

ALTEC (2009). Anales del XIII Seminario de la Asociación Latino-lberoamericana de Gestión Tecnológica. Ediciones Tecnológica de Bolívar: Cartagena de Indias.

ALTEC (2008). Anales del XII Seminario de la Asociación Latino-lberoamericana de Gestión Tecnológica.ALTEC, Buenos Aires.

ALTEC (2005). Anales del XI Seminario de la Asociación Latino-lberoamericana de Gestión Tecnológica. ALTEC, Salvador.

ALTEC (2003).Anales del X Seminario de la Asociación Latino-lberoamericana de Gestión Tecnológica. ALTEC, México D.F.

ALTEC (200I). Anales del IX Seminario de la Asociación Latino-lberoamericana de Gestión Tecnológica. ALTEC, San José.

ALTEC (1999). Anales del VIII Seminario de la Asociación Latino-lberoamericana de Gestión Tecnológica. ALTEC,Valencia.

ALTEC (1997). Anales del VIII Seminario de la Asociación Latino-lberoamericana de Gestión Tecnológica. ALTEC, La Habana.

ALTEC (1995). Anales delVI Seminario de la Asociación Latino-lberoamericana de Gestión Tecnológica. ALTEC, Concepción.

ALTEC (1993).Anales delV Seminario de la Asociación Latino-lberoamericana de Gestión Tecnológica. ALTEC, Bogotá.

ALTEC (1991). Anales del IV Seminario de la Asociación Latino-lberoamericana de Gestión Tecnológica. ALTEC, Caracas.

ALTEC (1989). Anales del III Seminario de la Asociación Latino-Iberoamericana de Gestión Tecnológica. ALTEC, Buenos Aires.

ALTEC (1987).Anales del II Seminario de la Asociación Latino-lberoamericana de Gestión Tecnológica. ALTEC, México D.F. 
ALTEC (1985). Anales del I Seminario de la Asociación Latino-lberoamericana de Gestión Tecnológica. ALTEC, São Paulo.

Galante, O. y Perez, N. (2008). Reseña Histórica de la Asociación Latino-iberoamericana de Gestión Tecnológica. ALTEC, Buenos Aires.

Quirino T., Baia, M., Rodrigues, I. y Sbragia, R. (200I). Innovación tecnológica, su política y gestión en el mundo ibérico: Cómo se refleja la disciplina en el Congreso de la ALTEC. Espacios.Vol. 22 (3).

Sbragia, R., Rodrigues, I. Baiao, M., Rego, T. (2003). Política e Gestão da Inovação Tecnológica: Estudo Comparativo da Evolução da Disciplina no Brasil e na lbero-América. Anales del $X$ Seminario de la Asociación Latino-lberoamericana de Gestión Tecnológica. ALTEC, México D.F. 
J.Technol. Manag. Innov. 2013,Volume 8, Special Issue ALTEC. 